\title{
Observability Analysis for Mobile Robot Localization
}

\author{
Agostino Martinelli and Roland Siegwart \\ Autonomous System Laboratory (EPFL) \\ Lausanne, Switzerland \\ Email: firstname.lastname@epfl.ch
}

\begin{abstract}
In this paper the problem of localize two mobile robots is considered. The robots are equipped with proprioceptive sensors (like encoders) and exteroceptive sensors able to provide relative observations between them. In these observations, one robot detects and identifies the other one and measures some relative quantity. An observability analysis is performed by taking into account the system nonlinearities and for four different relative observations. The theoretical results are validated by simulations and experiments carried out on real platforms. In these experiments, an Extended Kalman Filter is adopted to fuse the information coming from the encoders and the sensors performing the observations.
\end{abstract}

\section{INTRODUCTION}

Most of the localization methods have been developed for applications involving a single robot. Current research investigates applications where a team of robots collaborates to fulfill a mission. Single-robot localization approaches are not optimized to estimate the positions of all members of a team of collaborating robots. Indeed, an optimal strategy must take advantage of relative observations (detection of other robots). In [12], an Extended Kalman Filter (EKF) is used to fuse proprioceptive and exteroceptive sensor data. The equations of this filter are written in a decentralized form, allowing the decomposition into a number of smaller communicating filters. The approach relies on a particular relative observation, that is the relative configuration (position and orientation) between two robots. Experiments with a group of three robots successfully validated the method.

Localization is an estimation problem. The first issue to be addressed in any estimation problem is the observability property of the system. In control theory, a system is defined as observable when it is possible to reconstruct its initial state by knowing, in a given time interval, the control inputs and the outputs [7]. Usually, for the localization problem, the control inputs are directly estimated by the encoders and the outputs are the observations, as previously defined. The observability property has a very practical meaning. It is easy to realize from the definition that when a system is observable it contains all the necessary information to perform the estimation with an error which is bounded [7]. Regarding the localization problem, this means that the observability implies a bound error in the localization. The value of this bound obviously depends on the accuracy of the sensors. Regarding the localization problem, the observability analysis was carried out from several authors. Roumeliotis [12] presented it for a multi robots system equipped with encoder and sensors able to provide an observation consisting of the relative configuration between each pair of robots. The analysis was performed through the linear approximation. The main result of this observability analysis was that the system is not observable and it becomes observable when at least one of the robots in the team has global positioning capabilities. Bonnifait and Garcia considered the case of one robot equipped with encoders and sensors able to provide the bearing angles of known landmarks in the environment [3]. The observability analysis was carried out by linearizing the system (as in the previous case) and by applying the observability rank condition introduced by Hermann and Krener in [6] for nonlinear systems. As in many nonlinear systems, they found that in some cases while the associated linearized system is not observable, the system is observable. Bicchi and collaborators extended this result to the SLAM problem ([2], [9]). They considered one robot equipped with the same bearing sensors of the previous case. They considered in the environment landmarks with a priori known position and landmarks whose position has to be estimated. They found that two landmarks are necessary and sufficient to make the system observable. Furthermore, they applied optimal control methods in order to minimize the estimation error. In particular, in [9] they maximized the Cramer-Rao lower bound as defined in [8].

In this paper we consider the case of two robots in an environment where there isn't any landmark. We assume that both the robots are equipped with exteroceptive sensors able to provide relative observations between them. In these observations, one robot detects and identifies the other one and measures some relative quantity. This quantity will depend on both the robot configurations. We carry out the observability analysis for four different observations: the bearing of the second robot in the reference of the first robot $\left(B_{12}\right)$, the bearing of the first robot in the reference of second one $\left(B_{21}\right)$, the distance between the robots $(D)$ and the difference between the two absolute orientations $(O)$. These four observations contain the same information in the observation considered in [12]. In section II we define our system and the previous four observations. In section III we carry out the observability analysis based on the observability rank condition introduced by Hermann and Krener in [6] for nonlinear system. In IV we validate our theoretical results through simulations and real experiments carried out on real platforms. We adopt an $E K F$ to 
fuse the information coming from the encoders and the sensors performing the observations. Finally, conclusions are provided in section $\mathrm{V}$.

\section{THE SYSTEM}

We consider two mobile robots in a $2 D$-environment. The configuration of this system can be characterized through the vector $X=\left[x_{1}, y_{1}, \theta_{1}, x_{2}, y_{2}, \theta_{2}\right]^{T}$ containing the cartesian absolute coordinates of both the robots and their absolute orientations. The dynamics of this configuration is described through a non-linear differential equation $\dot{X}=f(X, u)$ where $u$ is the input control. We assume that both the robots have a differential drive system. In this case, $u=\left[v_{R_{1}}, v_{L_{1}}, v_{R_{2}}, v_{L_{2}}\right]^{T}$ where $v_{R_{i}}$ and $v_{L_{i}}$ are respectively the right and left wheel velocities for each robot $(i=1,2)$. Usually, $u$ is estimated during the navigation at a very high frequency through the encoders. The analytical expression for the function $f$ is:

$$
\begin{aligned}
& \dot{x}_{i}=v_{i} \cos \theta_{i} \\
& \dot{y}_{i}=v_{i} \sin \theta_{i} \quad i=1,2 \\
& \dot{\theta}_{i}=\omega_{i}
\end{aligned}
$$

where

$$
v_{i}=\frac{v_{R_{i}}+v_{L_{i}}}{2} \quad \omega_{i}=\frac{v_{R_{i}}-v_{L_{i}}}{d_{i}} \quad i=1,2
$$

and $d_{i}$ is the distance between the wheels for the $i^{t h}$ mobile robot.

Our robots are equipped with one or more exteroceptive sensors able to provide relative observations between them:

$$
y=h(X)
$$

We will consider the following four relative observations (see fig. 1):

- the bearing of the second robot in the reference of the first one ( $B_{12}$ in fig. 1);

- the bearing of the first robot in the reference of the second one $\left(B_{21}\right)$;

- the relative distance $(D)$;

- the relative orientation $(O$ i.e. the orientation of the second robot in the reference of the first one which is the same of the orientation of the first robot in the reference of the second one a part the sign).

The previous four observations are chosen because of the following two reasons:

- they can be easily implemented on real platforms with good accuracy (in particular, for the bearing angle, a camera can be used).

- when $B_{i j}, D$ and $O$ are simultaneously combined together they contain all the necessary information to estimate the configuration of the robot $j$ in the reference of the other robot (this global observation is the one considered in [12]);

In order to have simple expressions for the observation function $h$ for the previous four cases, it is very convenient

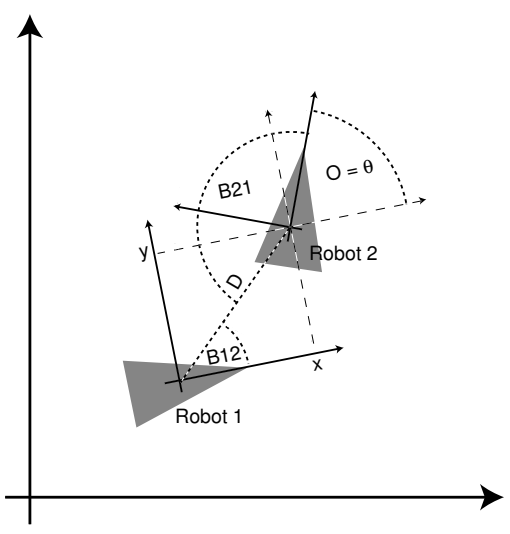

Fig. 1. The four considered observations

to adopt different parameters to characterize the configuration of the system. Indeed, the observability analysis based on the observability rank condition introduced by Hermann and Krener [6] for nonlinear systems, becomes dramatically easier if the expression of the function $h$ is simpler.

First of all, since the considered observations depend on the relative robot configurations, it is convenient to express the system configuration in terms of the absolute configuration of one robot (for instance the first) and the configuration of the other robot in the reference of the former. In other words, the new configuration will be $X_{n}=\left[x_{1}, y_{1}, \theta_{1}, x, y, \theta\right]$, where the last three components are defined by the following expressions (see also fig 1):

$$
\begin{array}{cc}
x= & \cos \theta_{1}\left(x_{2}-x_{1}\right)+\sin \theta_{1}\left(y_{2}-y_{1}\right) \\
y= & -\sin \theta_{1}\left(x_{2}-x_{1}\right)+\cos \theta_{1}\left(y_{2}-y_{1}\right) \\
\theta= & \theta_{2}-\theta_{1}
\end{array}
$$

Finally, it is convenient to introduce polar coordinates for the relative state $[x, y, \theta]$, namely:

$$
\begin{aligned}
& \rho=\sqrt{x^{2}+y^{2}} \\
& \phi=\operatorname{atan} 2(y, x)
\end{aligned}
$$

By adopting the changes in (4) and in (5) the system configuration is characterized through the state $R=$ $\left[x_{1}, y_{1}, \theta_{1}, \rho, \phi, \theta\right]^{T}$ and the dynamics obtained from (1) is:

$$
\begin{array}{cc}
\dot{x}_{1}= & v_{1} \cos \theta_{1} \\
\dot{y}_{1}= & v_{1} \sin \theta_{1} \\
\dot{\theta}_{1}= & \omega_{1} \\
\dot{\rho}= & v_{2} \cos \Delta-v_{1} \cos \phi \\
\dot{\phi}= & \frac{v_{2}}{\rho} \sin \Delta-\frac{v_{1}}{\rho} \sin \phi \\
\dot{\theta}= & \omega_{2}-\omega_{1}
\end{array}
$$

where $\Delta=\theta-\phi$.

In these coordinates the analytical expression for the four observations becomes:

$$
\begin{aligned}
& y_{O}=h(X)=\theta \\
& y_{D}=h(X)=\rho
\end{aligned}
$$




$$
y_{B_{12}}=h(X)=\phi
$$

and

$$
y_{B_{21}}=h(X)=\operatorname{atan} 2(\sin \Delta, \cos \Delta)
$$

\section{OBSERVABILITY ANALYSIS}

We consider separately the four systems defined by the same dynamics in (6) and one of the observation among the four in (7-10). For each system we individuate the observable part, namely the largest subsystem having the local distinguishability property as defined by Hermann and Krener in [6]. In particular, we determine the dimension $D_{O b s}$ of this subsystem and the dependence of $D_{O b s}$ on the considered input controls (which means to compute $D_{\text {Obs }}$ for the system when we inhibit one or several input controls). This kind of analysis was performed also in [3].

Note that a system contains the information to bound the error on the robot configuration only for its observable part. Since our systems are defined by changing the observation for the same dynamics, this analysis allows to choose an exteroceptive sensor instead of another one. Indeed, the sensor providing the observation whose corresponding system has the largest $D_{O b s}$, is preferable.

The dynamics in (6) is affine in the input variables, i.e. the state $R=\left[x_{1}, y_{1}, \theta_{1}, \rho, \phi, \theta\right]^{T}$ satisfies:

$$
\dot{R}=\sum_{k=1}^{4} f_{k}(R) u_{k}
$$

with $u_{1}=v_{1}, u_{2}=\omega_{1}, u_{3}=v_{2}, u_{4}=\omega_{2}$ and

$$
\begin{gathered}
f_{1}(R)=\left[\cos \theta_{1}, \sin \theta_{1}, 0,-\cos \phi,-\frac{1}{\rho} \sin \phi, 0\right]^{T} \\
f_{2}(R)=[0,0,1,0,0,-1]^{T} \\
f_{3}(R)=\left[0,0,0, \cos \Delta, \frac{\sin \Delta}{\rho}, 0\right]^{T} \\
f_{4}(R)=[0,0,0,0,0,1]^{T}
\end{gathered}
$$

We remind some concepts in the theory by Hermann and Krener in [6]. We will adopt the following notation. We indicate the $K^{\text {th }}$ order Lie derivative of a field $\psi$ along the vector fields $v_{i_{1}}, v_{i_{2}}, \ldots, v_{i_{K}}$ with $L_{v_{i_{1}}, v_{i_{2}}, \ldots, v_{i_{K}}}^{K} \psi$. Note that the Lie derivative is not commutative. In particular, in $L_{v_{i_{1}}, v_{i_{2}}, \ldots, v_{i_{K}}}^{K} \psi$ it is assumed to differentiate along $v_{i_{1}}$ first and along $v_{i_{K}}$ at the end. Let us indicate with $\Omega$ the space containing all the Lie derivatives $\left.L_{f_{i_{1}}, f_{i_{2}}, \ldots, f_{i_{K}}}^{K} h(X)\right|_{t=0}$ $\left(i_{1}, \ldots, i_{K}=1,2,3,4\right.$ and the functions $f_{i_{j}}$ are defined in (12-15)). Furthermore, we denote with $d \Omega$ the space spanned by the gradients of the elements of $\Omega$. In this notation, the observability rank condition can be expressed in the following way: The dimension $D_{O b s}$ at a given $X_{0}$ is equal to the dimension of $d \Omega$. Finally, we will indicate with $\Omega_{g}$ the space containing all the Lie derivatives whose order is less than or equal to $g$ and with $d \Omega_{g}$ the space spanned by the gradients of the elements of $\Omega_{g}$.

Before considering separately the four systems, we observe that the four observations in (7-10) only depend on the last three components of $R$. Furthermore, the four vector fields in (12-15) have the last three components only dependent on the same last three components of $R$. This means that the first three components of $R$ cannot be observed in anyone of our four systems. In particular, we can restrict our analysis to the observability of the last three components of $R$ which are: $\rho, \phi, \theta$. Therefore, since now, the gradients of the element of $\Omega$ are computed by differentiating only with respect to $\rho, \phi, \theta$ and instead of the vector fields in (12-15) we consider the following ones obtained by removing the first three components:

$$
\begin{gathered}
f_{1}(R)=\left[-\cos \phi,-\frac{\sin \phi}{\rho}, 0\right]^{T} \quad f_{2}(R)=[0,0,-1]^{T} \\
f_{3}(R)=\left[\cos \Delta, \frac{\sin \Delta}{\rho}, 0\right]^{T}
\end{gathered}
$$

We removed also $f_{4}(R)$ since is equal to $-f_{2}(R)$. We consider now the four systems separately.

\section{A. Relative Orientation}

The observation is defined in (7). Let us compute the Lie-derivatives along the three vector fields in (16).

We have:

$$
\begin{gathered}
L^{0} h=\theta \\
L_{f_{1}}^{1} h=0 \quad L_{f_{2}}^{1} h=-1 \quad L_{f_{3}}^{1} h=0
\end{gathered}
$$

Since they are constant, all the subsequent Lie derivatives are equal to 0 . In this case the space $d \Omega$ will be the space spanned by the vector $d L^{0} h=[0,0,1]^{T}$, and therefore $D_{O b s}=1$. In particular, this result is independent of the considered input controls (i.e. on the vector fields in (16)).

\section{B. Relative Distance}

From (8) we obtain $h=\rho$. Let us compute the elements of the associated $\Omega_{0}$. We have

$$
L^{0} h=\rho
$$

and the space $d \Omega_{0}$ will be the space spanned by the vector

$$
w_{1}=[1,0,0]^{T}
$$

The first order Lie derivatives are:

$$
L_{f_{1}}^{1} h=-\cos \phi \quad L_{f_{2}}^{1} h=0 \quad L_{f_{3}}^{1} h=\cos \Delta
$$

The space $d \Omega_{1}$ is spanned by the vectors:

$$
w_{2}=[0, \sin \phi, 0]^{T} \quad w_{3}=[0, \sin \Delta,-\sin \Delta]^{T}
$$

and $w_{1}$.

It is easy to realize that the dimension of this space is three. Furthermore, by computing only the Lie derivatives 
along $f_{2}(R)$ (i.e. by inhibiting both the tangential velocities), we get $D_{O b s}=1$.

Let us consider the case when only one of the two tangential velocities is inhibited. Note that, due to the symmetry of the distance with respect to the change Robot $1 \leftrightarrow$ Robot $2, D_{\text {Obs }}$ does not depend on the chosen velocity.

If we consider only $v_{1} \neq 0$ (i.e. we compute the Lie derivatives only along the vector $f_{1}(R)$ ) we obtain $D_{O b s}=$ 2 . Indeed, the space $\Omega$ will contain only function of $\rho$ and $\phi$.

By concluding, we found that $D_{O b s}=1$ when only the angular velocities are considered, $D_{O b s}=2$ when one of the two tangential velocities is considered and $D_{\text {Obs }}=3$ if both $v_{1}$ and $v_{2}$ are different from 0 .

\section{Relative Bearing}

In contrast to the previous cases, the bearing observation changes with the change Robot $1 \leftrightarrow$ Robot2. In particular, we have the two distinct expressions given respectively in (9) and (10). On the other hand, the dimension $D_{O b s}$ obtained by considering $B_{i j}$ and some control $p$ for the robot $i$ and some control $q$ for the robot $j$ is the same obtained by considering $B_{j i}$ and the control $p$ for the robot $j$ and the control $q$ for the robot $i$. Therefore, we can restrict the analysis by only considering the bearing $B_{12}$ in (10) and the three vector fields in (16).

We have

$$
L^{0} h=\phi
$$

and the space $d \Omega_{0}$ will be the space spanned by the vector

$$
w_{1}=[0,1,0]^{T}
$$

The first order Lie derivatives are:

$$
L_{f_{1}}^{1} h=-\frac{\sin \phi}{\rho} \quad L_{f_{2}}^{1} h=0 \quad L_{f_{3}}^{1} h=\frac{\sin \Delta}{\rho}
$$

The space $d \Omega_{1}$ is spanned by the vectors:

$w_{2}=\left[\frac{\sin \phi}{\rho^{2}},-\frac{\cos \phi}{\rho}, 0\right]^{T} w_{3}=\left[-\frac{\sin \Delta}{\rho^{2}},-\frac{\cos \Delta}{\rho}, \frac{\cos \Delta}{\rho}\right]^{T}$

and $w_{1}$.

It is easy to realize that the dimension of this space is three. Furthermore, by computing only the Lie derivatives along $f_{2}(R)$ (i.e. by inhibiting both the tangential velocities), we get $D_{O b s}=1$.

If we consider only $v_{1} \neq 0$ (i.e. we compute the Lie derivatives only along the vector $f_{1}(R)$ ) we obtain $D_{O b s}=$ 2 as in the previous case. On the other hand, if we consider $v_{2} \neq 0$ (i.e. we compute the Lie derivatives only along the vector $f_{3}(R)$ ) it is possible to verify that $D_{O b s}=3$ (provided that $\theta \neq \phi$ ).

We conclude this section by observing that the bearing angle contains more useful information than the other observations. This is due not only to the fact that in this case it exists one velocity able alone to make $D_{O b s}=3$ but also because if each robot is equipped with sensors able to

\begin{tabular}{|c|l|l|l|l|c|}
\hline Controls $\neq 0$ & $O$ & $D$ & $B_{12}$ & $B_{21}$ & $B_{12}+B_{21}$ \\
\hline anyone & 1 & 1 & 1 & 1 & 2 \\
$v_{1}$ & 1 & 2 & 2 & 3 & 3 \\
$\omega_{1}$ & 1 & 1 & 1 & 1 & 2 \\
$v_{2}$ & 1 & 2 & 3 & 2 & 3 \\
$\omega_{2}$ & 1 & 1 & 1 & 1 & 2 \\
$v_{1} \& v_{2}$ & 1 & 3 & 3 & 3 & 3 \\
All & 1 & 3 & 3 & 3 & 3 \\
& & & & & \\
\hline
\end{tabular}

TABLE I

DiMENSION OF THE OBSERVABLE PART OF THE SYSTEM ( $D_{\text {Obs }}$ ) VS THE INPUT CONTROLS DIFFERENT FROM 0

provide the bearing angle of the other one, the two distinct measurements contain some complementary information. In particular, following similar computation, it is possible to verify that the dimension $D_{O b s}$ of the system defined with both the observations $B_{12}$ and $B_{21}$ is always larger or equal to 2 (even when all the controls are inhibited) and becomes 3 as soon as one of the two tangential velocities is set different from zero.

The results of this section are resumed in table I.

\section{RESULTS}

We validated the previous theoretical results through simulations (sect. IV-A) and experiments carried out on real platforms (sect. IV-B). As stated in section II, in IV-A and in IV-B our robots are equipped with sensors able to estimate the control $u$ (encoder sensors) and to perform the observations defined in II. Actually, in the real experiments, we only consider the two type of bearings $\left(B_{21}\right.$ and $\left.B_{12}\right)$.

We adopted an Extended Kalman Filter $(E K F)$ to fuse the information coming from the encoders and the observations. The equations of this filter applied to the multi robot localization can be found in [11].

\section{A. Simulations}

We performed several $M A T L A B$ simulations by considering different types of robot trajectory. Although in a simulation the unities are not important in an absolute sense, we adopted the values we get from previous experiments in our lab to be close to the reality (e.g. see [1] and [10]). The entire robot motion takes $200 \mathrm{sec}$. The encoders data are delivered at $100 \mathrm{~Hz}$ while the observations data at $1 \mathrm{~Hz}$ for all the considered four observations.

The error on the encoder data are characterized with the model introduced by Chong and Kleeman for a differential drive system [5], where the error in the wheel displacements is assumed gaussian with a variance increasing linearly with the distance travelled by each wheel. In particular, we set the parameter characterizing this proportionality equal to the value we found through experiments in our lab, [10], i.e. $510^{-5} \mathrm{~m}$.

Regarding the observations, we adopted the following variances to characterize the error (assumed to be zero mean with gaussian distribution and variance $R$ ) for the 


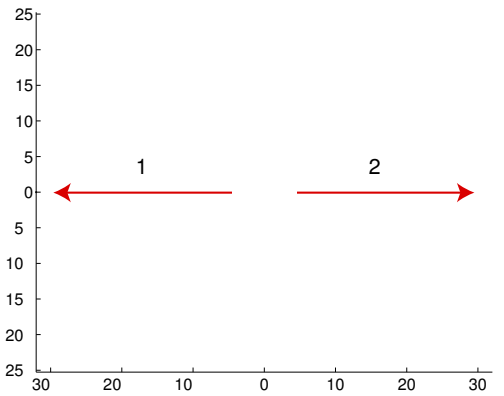

Fig. 2. The simulated robot motions for the two robots

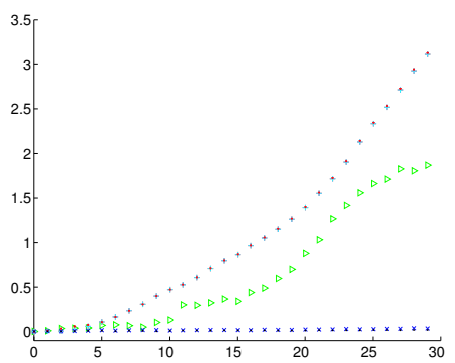

Fig. 3. The error for the estimated position vs the distance travelled by each robot when the estimation is carried out with only odometry (red .), fusion of odometry with the relative distance (green $\triangleright$ ), fusion of odometry with the relative orientation (sky-blue + ), fusion of odometry with both the relative bearings (blue $x$ ) and fusion of odometry with all the relative observations together (black .). The plotted error is averaged over the two robots.

four considered observations: $R=\sigma_{D}^{2}=(0.02 m)^{2}$ and $R=\sigma_{O}^{2}=\sigma_{B_{12}}^{2}=\sigma_{B_{21}}^{2}=(1 \mathrm{deg})^{2}$.

Figure 3 illustrates the results for the simulated experiment with the two robots moving along the straight lines in fig 2 . We conclude that the accuracy on the localization is strongly improved by using the relative bearing. The result obtained by fusing all the relative observations with odometry is slightly better than the one obtained by fusing only both the relative bearings with the odometry (in particular the final position error after $30 \mathrm{~m}$ of navigation is $0.0175 \mathrm{~m}$ when all the relative observations are integrated and $0.0356 \mathrm{~m}$ when only the relative bearings are adopted).

Regarding other robot trajectories, similar results can be obtained when one robot in the team perform pure rotations (i.e. $v_{R}=-v_{L}$ ) and the other one moves along a straight line. On the other hand, for more general robot trajectories (obtained by setting at each time step the value of $v_{R}$ and $v_{L}$ randomly), the results can be sometimes different. Indeed, the relative bearing usually shows slightly better performances with respect to the other relative observations but it is not always the case. However, simulations tend to indicate that the relative bearing clearly outperforms other relative observations whenever the robot trajectories are similar as the one presented on figure 2 .

\section{B. Real Experiments}

For the real experiments we adopted the smartease robots [13] equipped with encoders and linear camera. This second sensor was adopted to get the bearing angle of the

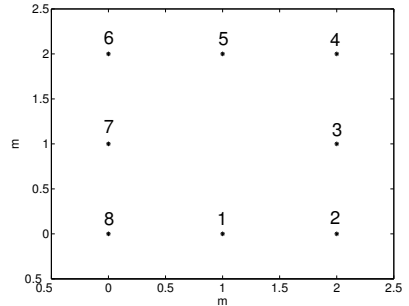

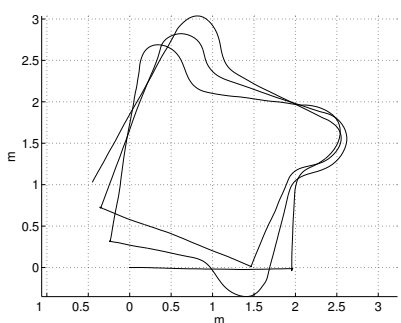

$b$
Fig. 4. $a$ : The real experiment in the case of one moving robot. The motion of the second robot consisted of three loops passing over the indicated points while the first robot was standing at the origin. $b$ : The first set of experiments consisting only of one trial. The robot position is estimated using only the encoder data.

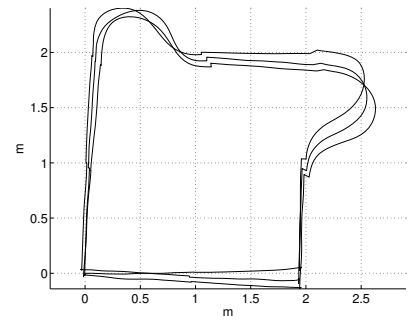

$a$

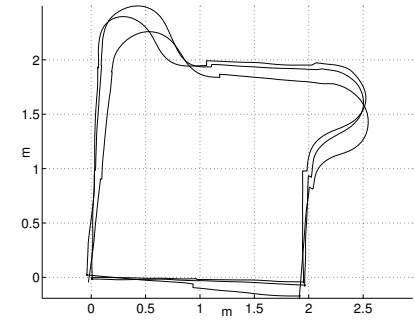

$b$
Fig. 5. Second set of experiments, first trial $(a)$ and second trial $(b)$. The robot position of the moving robot is estimated by fusing the encoder data with the data coming from the bearing of the robot standing at the origin in the reference of the other one $\left(B_{21}\right)$ through an $E K F$.

other robot. We performed two types of experiments. In the former, only the second robot was moving while the first one was standing at the origin of the reference frame, in the latter both the robots were moving simultaneously.

1) Moving only the second robot: We performed three sets of experiments. In the first experiment we only used the odometry to localize the moving robot. In the second set of experiments, we fused the encoder data with the observation data consisting of the bearing $B_{21}$ (i.e. the bearing of the robot at the origin in the reference of the moving robot). Finally, in the third one, the observation consisted of the bearing of the moving robot in the reference of the robot at the origin $\left(B_{12}\right)$. We moved the robot along a trajectory passing through the points indicated in fig. $4 a$. Each experiment consisted of three loops passing through these points. The observations were performed when the robot was standing on the previous points.

In figures $4 b-6$ we have to consider the estimated robot position only in the points displayed in fig. $4 a$. Indeed, the ground truth is only almost known there. In the other points, the real robot trajectory is different for any loop and for any trial. We conclude that, while the estimation error becomes divergent in the case of the $B_{21}$ it is constant in the case of $B_{12}$, accordingly with the theory. Indeed, the theory proved that in the first case $D_{O b s}=2$ while in the second one $D_{O b s}=3$. Since one robot does not move, the dimension of the space to be estimated is 3 and therefore only in the second case this space is observable. 


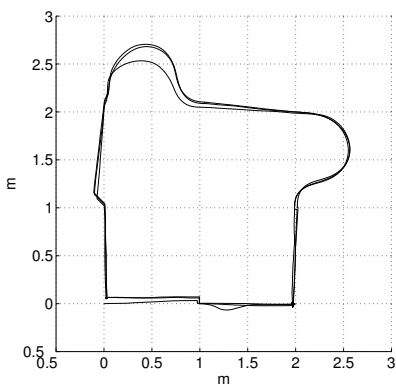

$a$

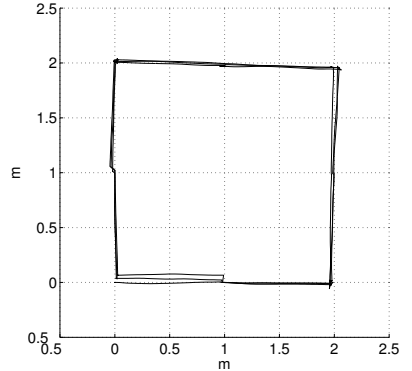

$b$
Fig. 6. Third set of experiments, first trial $(a)$ and second trial $(b)$. The robot position of the moving robot is estimated by fusing the encoder data with the data coming from the bearing of the moving robot in the reference of the other one $\left(B_{12}\right)$ through an $E K F$.

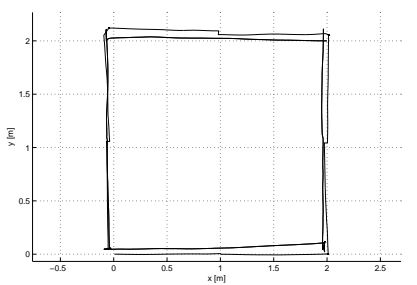

$a$

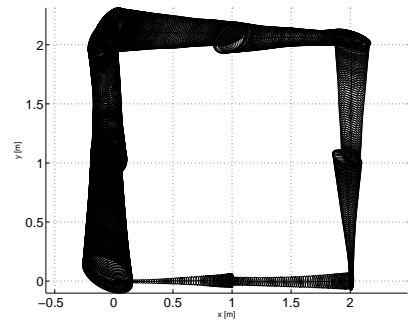

$b$
Fig. 7. The experiment with both the robots moving. $a$ The estimated robot trajectories, blue for the first robot, red for the second one. $b$ The estimated position error for the first robot during the motion.

2) Moving both the robots simultaneously: The initial robot configurations are respectively for the first and the second robot, $[2,0,0]$ and $[0,2, \pi]$. The robots moved simultaneously along the same trajectory represented in fig. $4 a$ (counterclockwise for both the robots), being the starting points the $8^{t h}$ and the $4^{t h}$ respectively for the first and the second robot. Each robot made one loop. The relative observations (consisting of both $B_{12}$ and $B_{21}$ ) occurred each meter of navigation. In fig. $7 a$ we plot the estimated robot trajectories and in fig. $7 b$ we show the estimated position error for the first robot during the motion. In agreement with the theory, the error in this case is not bounded. Indeed, the configuration of the system has 6 independent parameters while $D_{O b s}=3$. Anyway, the error reduces in correspondence of each relative observation.

\section{CONCLUSION}

In this paper we considered the problem of localize simultaneously two mobile robots able to perform relative observations among them and equipped with proprioceptive sensors like encoders. We carried out an observability analysis based on the Observability rank Condition introduced by Hermann and Krener in [6] for nonlinear systems. This analysis showed us the relative bearing as the best observation between the robots. Indeed, the part of the system which is observable is in general larger than for the other relative observations (relative distance and relative orientation).
Several simulations and experiments carried out on a real platform validated our theoretical results. In these experiments, an Extended Kalman Filter was adopted to fuse the information coming from the encoder and the observation data.

Currently, we are considering the case of a mobile robot whose odometry is not calibrated. In this case, the observability analysis will extend to the parameters characterizing the odometry error (e.g. wheel diameters, distance between the wheel). It would be interesting to find optimal trajectories as in [9] in order to minimize the error on the estimation of these parameters. In this way, it would be possible to obtain a natural generalization of the UMBmark method introduced by Borenstein and collaborators [4].

\section{ACKNOWLEDGMENT}

This work has been supported by the European project RECSYS (Real-Time Embedded Control of Mobile Systems with Distributed Sensing) and the Swiss project $F N$ (Scheizerischer Nationalfonds zur Forderung der Wissenschaftlichen Scientifique). The authors would like also to thank Niklaus Hugi for the implementations on the real platform and Antonio Bicchi and Ruggero Frezza for helpful discussions.

\section{REFERENCES}

[1] K.O.Arras, N.Tomatis, B.T.Jensen and R.Siegwart, "Multisensor on-the-fly localization: Precision and reliability for applications", Robotics and Autonomous Systems 34, pp. 131-143, 2001.

[2] Bicchi A., Pratichizzo D., Marigo A. and Balestrino A., On the Observability of Mobile Vehicles Localization, IEEE Mediterranean Conference on Control and Systems, 1998

[3] Bonnifait P. and Garcia G., Design and Experimental Validation of an Odometric and Goniometric Localization System for Outdoor Robot Vehicles, IEEE Transaction On Robotics and Automation, Vol 14, No 4, August 1998

[4] Borenstein J., Feng L., "Measurement and correction of systematic odometry errors in mobile robots," IEEE Transactions on Robotics and Automation, vol. 12, pp. 869-880, 1996.

[5] Chong K.S., Kleeman L., "Accurate Odometry and Error Modelling for a Mobile Robot," International Conference on Robotics and Automation, vol. 4, pp. 2783-2788, 1997.

[6] Hermann R. and Krener A.J., 1977, Nonlinear Controllability and Observability, IEEE Transaction On Automatic Control, AC-22(5): 728-740

[7] Isidori A., Nonlinear Control Systems, 3rd ed., Springer Verlag, 1995.

[8] Kosut R.L., Arbel A. and Kessler K.M., Optimal Sensor System Design for State Reconstruction, IEEE Transaction On Automatic Control, Vol 27, No 1, February 1982

[9] Lorussi F, Marigo A. and Bicchi A., Optimal exploratory paths for a mobile rover, IEEE International Conference on Robotics and Automation (ICRA), 2001, Vol 2, Pages:2078 - 2083

[10] Martinelli A, Tomatis N, Tapus A. and Siegwart R., "Simultaneous Localization and Odometry Calibration" International Conference on Inteligent Robot and Systems (IROSO3) Las Vegas, USA

[11] Martinelli A., Pont F. and Siegwart R., "Multi-Robot Localization Using Relative Observations" International Conference on Robotics and Automation, April 2005, Barcellona, Spain.

[12] S.I. Roumeliotis and G.A. Bekey, 2002, Distributed Multirobot Localization, IEEE Transaction On Robotics and Automation Vol 18, No.5, October 2002

[13] http://www.smartrob.org, http://asl.epfl.ch/contests/current/resources.shtml 